in vivo $34: 2159-2162(2020)$

doi:10.21873/invivo.12023

\title{
A Case of Metastatic Iris Tumor Observed With Anterior Segment Optical Coherence Tomography Before and After Radiation Therapy
}

\author{
MIZUHO MITAMURA $^{1,2}$, SATORU KASE $^{1}$, YUKA SUIMON $^{1}$, TAKAKO ITO $^{1}$ and SUSUMU ISHIDA ${ }^{1}$ \\ ${ }^{1}$ Department of Ophthalmology, Faculty of Medicine and Graduate School of Medicine, \\ Hokkaido University, Sapporo, Japan; \\ ${ }^{2}$ Department of Ophthalmology, Teine Keijinkai Hospital, Sapporo, Japan
}

\begin{abstract}
Background/Aim: Metastatic iris tumors are relatively rare entities that are often treated with radiation therapy; however, it remains difficult to evaluate the effectiveness of treatment. Anterior segment optical coherence tomography (AS-OCT) provides high-resolution crosssectional images with no dye injection, and may thus be useful for evaluating iris tumors. Herein, we report a case of metastatic iris tumor examined with AS-OCT before and after radiation therapy. Case Report: A 56-year-old Japanese woman complained of ocular hyperemia and pain in her right eye, and was referred to our hospital because of uveitis and an iris mass. One year before the initial visit, the patient was diagnosed with lung adenocarcinoma, and treated with chemotherapy. She also underwent radiation therapy 4 months ago due to multiple bone metastases. Slit-lamp microscopy revealed a $2 \times 2 \mathrm{~mm}$ elevated lesion with partial depigmentation on the lower iris. AS-OCT examination revealed that the mass was located in the iris stroma with an irregular anterior surface. Based on fine needle aspiration cytology, she was diagnosed with metastatic iris tumor derived from lung adenocarcinoma, leading to local radiotherapy. Seven months later, AS-OCT showed shrinkage and atrophy of the iris tumor with peripheral anterior synechia formation. Conclusion: We report a case of cytology-proven metastatic iris tumor observed with AS-OCT before and after radiation
\end{abstract}

This article is freely accessible online.

Correspondence to: Satoru Kase, MD, Ph.D., Department of Ophthalmology, Faculty of Medicine and Graduate School of Medicine, Hokkaido University, N-15, W-7, Kita-ku, Sapporo 0608638, Japan. Tel: +81 117065944, Fax: +81 117065948, e-mail: kaseron@med.hokudai.ac.jp

Key Words: Iris tumor, iris metastasis, anterior segment optical coherence tomography, aspiration cytology. therapy. This study highlights the potential usefulness of ASOCT for monitoring changes in tumor size and configuration following radiotherapy.

Uveal metastasis of systemic cancer takes place in the eye, accounting for $12 \%$ of all the malignancies, according to autopsy evaluations (1). Uveal metastases occur less frequently in the iris than in the choroid, possibly due to a difference in substantial blood supply via the posterior ciliary arteries (2). The rate of intraocular metastases varies based on the primary tumor site and the degree of systemic therapy. In the largest published series of 107 eyes with metastatic tumors of the iris, Shields et al. reported that the breast (33\%) was the most common site of the primary tumor, followed by lung (27\%), skin (12\%), kidney (7\%), esophagus (3\%), and other organs (3).

The common ocular symptoms of patients with iris metastasis are pain and blurred vision (4). However, some patients are likely to have no ocular symptoms even with advanced systemic cancer, thus leading to a lesser chance for ophthalmologists to encounter such patients than for oncologists and primary physicians. Regarding ophthalmological presentation, metastatic iris tumors can manifest as a unilateral mass, which may be associated with a variety of ocular complications, including glaucoma, abnormal iris vascularization, hyphema, ectropion uvea, pigment dispersion, pseudohypopyon, iridocyclitis and pupillary distortion (5).

The concept of treatment for uveal metastases, including iris metastasis, is palliative. The median time interval between the detection of iris metastasis and death is reportedly 24 months (6). Patients with iris metastasis should also be treated with systemic chemotherapy, while external radiotherapy or brachytherapy is recommended for the treatment of local metastasis (7). However, it is difficult to evaluate the therapeutic efficacy for iris metastasis. Anterior segment optical coherence tomography (AS-OCT) provides high-resolution cross-sectional images of anterior segment 

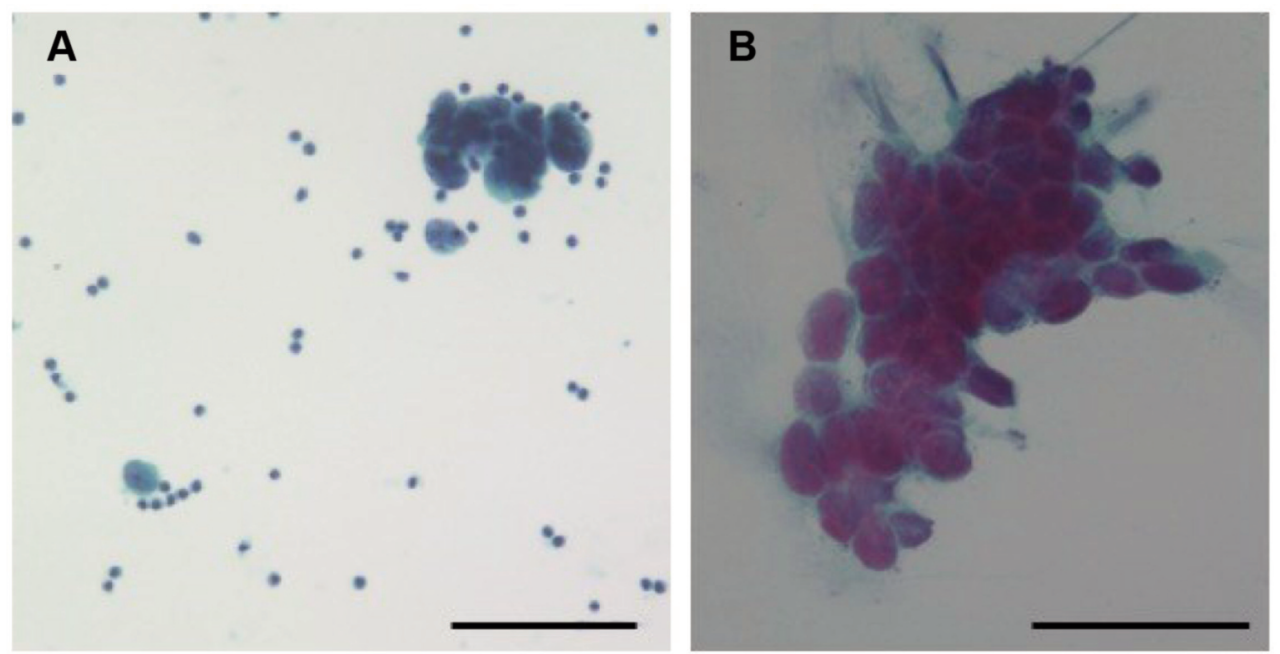

Figure 1. Cytological findings of the patient's pleural effusion and iris tumor. (A) Cytological specimen of pleural effusion. Small to large clumps consisting of small cells and atypical cells with an extremely high nucleo-cytoplasmic ratio were observed. The bar indicates $50 \mu \mathrm{m}$. (B) Cytological specimen of the iris tumor. Epithelial-like cells with an increased nucleo-cytoplasmic ratio were observed in small clusters, which showed dysplasia, distinct nucleoli, and irregularly shaped nuclei by Papanicolaou staining. The bar indicates $50 \mu \mathrm{m}$.

tissues including the iris with no dye injection. The use of AS-OCT has steadily increased over the last 10 years, and may contribute to the detailed demonstration of iris tumors. We herein present a case of metastatic iris tumor observed with AS-OCT before and after radiation therapy.

\section{Case Report}

A 56-year-old Japanese woman complained of ocular hyperemia and pain in her right eye, and was referred to our hospital because of uveitis and an iris mass. One year before the initial visit, the patient was diagnosed with lung adenocarcinoma complicated by cancerous pleurisy based on clinico-cytological examinations (Figure 1A). She received chemotherapy for two months until 6 months before the initial visit. Since she developed multiple bone metastases in her thoracolumbar spines, left ribs, and sternum, radiation therapy was also performed 4 months before the initial visit. Her smoking habit was two packs of cigarettes per day for 32 years. As for her family history, her father died of pancreatic cancer, and her mother and sister had a history of skin and breast cancers, respectively.

Her decimal best-corrected visual acuity (BCVA) was 1.0 oculi uterque (OU) with mixed astigmatism, and intraocular pressure was normal OU. Slit-lamp microscopy revealed an elevated lesion, measuring $2 \times 2 \mathrm{~mm}$, with partial depigmentation on the lower iris surface oculus dexter (OD) (Figure 2A and B). Anterior segment inflammation subsided with use of betamethasone eye drops, prescribed by her former ophthalmologist. Although posterior synechia was not observed, mydriasis was hampered by the elevated iris lesion
OD. AS-OCT (CASIA2, TOMEI Corp., Japan) was performed by an experienced examiner to image the same cross section that cuts the most center of the iris tumor. ASOCT examination revealed that the solid mass was located in the iris stroma with an irregular anterior surface, and did not extend to the ciliary body. While the anterior surface of the mass was defined by high reflectivity, its posterior surface boundary and the iris pigment epithelium could hardly be distinguished (Figure 2C). Fundus examination showed no obvious abnormalities OU.

We performed fine needle aspiration cytology of the iris mass. The procedure was as follows: a $27 \mathrm{G}$ needle was attached to a $1 \mathrm{cc}$ syringe. The needle was penetrated from the corneal limbs, the iris tumor tissue was directly punctured, and tumor cells were aspirated. There are few bleedings in the eye during the procedure. Papanicolaou staining was performed to evaluate morphological findings.

The aspiration cytology of the mass revealed nonpigmented, atypical epithelial cells with an increased nucleocytoplasmic ratio, irregular-shaped nuclei, and prominent nucleoli (Figure 1B). Since the cytological findings of the iris were morphologically consistent with those of her primary lung cancer (Figure 1A), she was diagnosed with metastatic iris tumor derived from lung adenocarcinoma. Magnetic resonance imaging also detected another metastatic lesion in the medulla oblongata.

In cooperation with physicians and radiologists, she underwent radiation therapy to the right eyeball and medulla oblongata, the dosage/fraction of which were $36 \mathrm{~Gy} / 12$ fraction (fr) and $39 \mathrm{~Gy} / 13 \mathrm{fr}$, respectively. After 7 months, she was well with no ocular hyperemia or pain. BCVA was 0.7 




Figure 2. Slit-lamp and AS-OCT findings before and after radiotherapy. (A) Slit-lamp microscopy showed a $2 \times 2$ mm elevated lesion with partial depigmentation on the lower iris surface OD. (B) Enlarged anterior segment photograph of the tumor. (C) AS-OCT examination revealed that the mass was located in the iris stroma with an irregular anterior surface. (D) Post-treatment images demonstrated atrophy of the lesion. (E) After radiotherapy, AS-OCT showed shrinkage of the iris tumor and thinning of the iris tissue.

with normal intraocular pressure OD. Slit-lamp examination showed atrophy of the iris tumor with flattening of its surface (Figure 2D). There was mild anterior segment inflammation with occasional cells and keratic precipitates, together with posterior synechia and peripheral anterior synechia (PAS) at 5-7 o'clock. AS-OCT showed shrinkage of the iris tumor and thinning of the iris tissue with PAS formation (Figure 2E).

\section{Discussion}

We encountered a case of metastatic iris tumor successfully treated by radiation therapy, which was monitored with ASOCT before and after treatment. In a clinical survey of 3,680 iris tumors, $21 \%$ of cases were morphologically cystic with the remaining $79 \%$ solid, which consisted of melanocytic tumors in $68 \%$ and non-melanocytic tumors in $11 \%$ (8). Non-melanocytic iris tumors have a variety of diagnoses including metastatic tumors, the frequency of which is only $2 \%$ (67 tumors) (8). Our present case appeared to be a solid mass with low inner reflectivity on AS-OCT, while cytology further proved the iris lesion as a non-melanocytic metastatic tumor.

It is likely that melanocytic malignant tumors such as melanoma arising from the ocular regions revealed more vascular-rich nature than non-malignant melanocytic tumors (9). In this study, in aspirating the iris tumor tissue for the diagnosis, intraoperative and postoperative bleeding was not apparent in the anterior chamber, suggesting that metastatic iris tumors might be characterized by hypovascular non-melanocytic tumors. Careful observation during the procedure might also contribute to the presumed diagnosis of the iris tumors.
Various imaging techniques such as transillumination, anterior segment photography, ultrasonic biomicroscopy (UBM), and indocyanine green angiography (ICGA) have been used to monitor iris tumors in previous studies (10). Transillumination and anterior segment photography only allow observation of the anterior aspect of tumors, whereas these methods have little or no capacity for evaluating the thickness or content of tumors. Similarly, ICGA would not be suitable for observing the size, thickness, and internal structure of tumors, even with an invasive procedure of intravenous dye injection.

UBM provides valuable information about the anterior segment of the eye because it has a high penetration strength, which enables extension of detectable region to the ciliary body. Moreover, UBM is unaffected by pigmentation, and allows clear imaging of the posterior margin of iris tumors. There have been many studies comparing AS-OCT with other anterior segment imaging modalities $(11,12)$. Pavlin et al. reported that AS-OCT was useful in small hypopigmented tumors limited to the iris, despite the superiority of UBM to AS-OCT in demonstrating highly pigmented and ciliary body tumors due to the high tissue penetration of UBM (11). Hau et al. compared UBM with AS-OCT in the examination of 126 eyes with iris or iridociliary body lesions, showing that the axial resolution was higher in AS-OCT than in UBM, together with the superiority of AS-OCT in visualizing lesions involving the lateral and anterior aspects of the iris (12). AS-OCT utilizes a wavelength of 1,310 nm for image acquisition, provides a higher image resolution, and measures less invasively than UBM. In our case, the metastatic tumor cytologically 
represented a non-pigmented epithelial tumor consistent with lung adenocarcinoma. AS-OCT clearly demonstrated the tumor configuration located in the iris stroma at the initial visit, and eventually the resolution of the mass following radiotherapy through repeated tests. In the present case, therefore, the high-resolution image quality and noncontact/noninvasive nature of AS-OCT would contribute to the detailed structural demonstration of the iris tumor and long-term follow-up with repeated imaging at the same lesion, respectively.

Unequivocally, radiologists perform radiotherapy for metastatic iris tumors $(7,13)$ and thus need to determine its efficacy. However, it would be difficult to monitor threedimensional anatomical changes by using conventional computed tomography and magnetic resonance imaging, because tumors are too small for these modalities. The advantage of AS-OCT imaging is likely to consist in its convenient usability for both radiologists and ophthalmologists to share vital information about morphologic changes as well as therapeutic effects.

In conclusion, we reported on a case of metastatic iris tumor observed by AS-OCT before and after radiation therapy. AS-OCT is useful for evaluating detailed anatomical findings and post-treatment sequential changes in the management of metastatic iris tumors.

\section{Conflicts of Interest}

The Authors declare no conflicts of interest regarding this study.

\section{Authors' Contributions}

MM corrected clinical data and wrote a whole paper. SK and YS acquired pathological findings and interpreted the clinicopathological data. SK, YS, TI and SI supervised the manuscript and did critical revision.

\section{References}

1 Bloch RS and Gartner S: The incidence of ocular metastatic carcinoma. Arch Ophthalmol 85(6): 673-675, 1971. PMID: 5562786. DOI: 10.1001/archopht.1971.00990050675005

2 Shields CL, Shields JA, Gross NE, Schwartz GP and Lally SE: Survey of 520 eyes with uveal metastases. Ophthalmology 104(8): 1265-1276, 1997. PMID: 9261313. DOI: 10.1016/s01616420(97)30148-1

3 Shields CL, Kaliki S, Crabtree GS, Peshatani A, Morton S, Anand RA, Coco G and Shields JA: Iris metastasis from systemic cancer in 104 patients: The 2014 Jerry A. shields Lecture. Cornea 34(1): 42-48, 2015. PMID: 25343701. DOI: 10.1097/ICO. 0000000000000285
4 Ferry AP and Font RL: Carcinoma metastatic to the eye and orbit II: A clinicopathological study of 26 patients with carcinoma metastatic to the anterior segment of the eye. Arch Ophthalmol 93(7): 472-482, 1975. PMID: 166633. DOI: 10.1001/archopht.1975.01010020488002

5 Kanthan GL, Jayamohan J, Yip D and Conway RM: Management of metastatic carcinoma of the uveal tract: An evidence-based analysis. Clin Exp Ophthalmol 35(6): 553-565, 2007. PMID: 17760639. DOI: 10.1111/j.1442-9071.2007.01550.x

6 Ferry AP and Font RL: Carcinoma metastatic to the eye and orbit. I: A clinic-pathological study of 227 cases: Arch Ophthalmol 92(4): 276-286, 1974. PMID: 4412321. PMID: 4412321. DOI: 10.1001/archopht.1974.01010010286003

7 Mello PC, Brasil OF, Vidoris A, Morales MC and Belfort RN: Iris metastases from systemic cancer: A report of three cases. Arq Bras Oftalmol 79(6): 407-410, 2016. PMID: 28076572. DOI: $10.5935 / 0004-2749.20160115$

8 Shields CL, Kancherla S, Patel J, Vijayvargiya P, Suriano MM, Kolbus E, Badami A, Sharma P, Jacobs E, Voluck M, Zhang Z, Kansal R, Shields PW, Bianciotto CG and Shields JA: Clinical Survey of 3680 iris tumor based on the age at presentation. Ophthalmology 119(2): 407-414, 2012. PMID: 22035581 DOI: 10.1016/j.ophtha.2011.07.059

9 Spiliopoulos K, Peschos D, Batistatou A, Ntountas I, Papoudou-Bai A, Zioga A, Agnantis N and Kitsos G: immunohistochemical study of vasculogenic mimicry and angiogenesis in melanocytic tumors of the eye and the periocular area. Anticancer Res 37(3): 1113-1120, 2017. PMID: 28314272. DOI: 10.21873 /anticanres.11424

10 Arslantürk M, Gündüz AK and Gündüz OO: Evaluation of iris melanoma with anterior segment optical coherence tomography. Turk J Ophthalmol 47(4): 231-234, 2017. PMID: 28845329. DOI: $10.4274 /$ tjo.66742

11 Pavlin CJ, Vásquez LM, Lee R, Simpson ER and Ahmed II: Anterior segment optical coherence tomography and ultrasound biomicroscopy in the imaging of anterior segment tumors. Am J Ophthalmol 147(2): 214-219, 2009. PMID: 18930449. DOI: 10.1016/j.ajo.2008.08.023

12 Hau SC, Papastefanou V, Shah S, Sagoo MS, Restori M and Cohen V: Evaluation of iris and iridociliary body lesions with anterior segment optical coherence tomography versus ultrasound B-scan. Br J Ophthalmol 99(1): 81-86, 2015. PMID: 25091953. DOI: 10.1136/bjophthalmol-2014-305218

13 Das C and Shields CL: Radiotherapy for iris metastasis from esophageal carcinoma: A series of three cases, Oman J Ophthalmol 9(2): 93-96, 2016. PMID: 27433035. DOI: 10.4103/0974-620X.184526
Received April 7, 2020

Revised April 17, 2020

Accepted April 18, 2020 